\title{
Auf dem Weg zu mehr Kollaboration: Kollaboratives Lernen als Ansatz der Lehrkräfteprofessionalisierung in der Grundbildung
}

\author{
Esther Winther (D) J Jessica Paeßens • Beifang Ma • Monika Tröster • \\ Beate Bowien-Jansen
}

Eingegangen: 1. Juli 2021 / Überarbeitet: 6. Oktober 2021 / Angenommen: 28. Oktober 2021 / Online publiziert: 26. November 2021

(C) Der/die Autor(en) 2021

Zusammenfassung Kollaborative Lehrkräfteprofessionalisierung ist eine wichtige Maßnahme, um Lehrkräfte zu befähigen, Herausforderungen der postpandemischen Arbeitsrealität zu bewältigen. Wie dies in der Lehrkräfteprofessionalisierung in der Grundbildung gelingt bzw. gefördert werden kann, ist bislang unzureichend erforscht. Dieser Beitrag nimmt sich dieses Desiderats an und beschreibt explorativ Gelingensbedingungen des kollaborativen Lernens im Rahmen eines Ausschnitts der kollaborativen Lehrkräfteprofessionalisierung. Hierbei wird das kollaborative Lernen entlang einer Lernspielvalidierung für die Finanzielle Grundbildung zum Gegenstand der Weiterbildung gemacht. Es zeigt sich, dass u.a. der Aufbau einer Teamorganisation, die Leitung eines Teams sowie die Aufgabenteilung innerhalb eines Teams relevant für das kollaborative Lernen von Lehrkräften sind und dass die Berufserfahrung die Beziehung zwischen der wahrgenommenen Wirksamkeit des Kollaborationsprozesses und der Entstehung des situationalen Interesses an Kollaboration moderiert.

Schlüsselwörter Kollaboratives Lernen · Lehrkräftefortbildung · Lernspiel · Mixed Methods · Finanzielle Grundbildung

Prof. Dr. Esther Winther $(\bowtie) \cdot$ Jessica Paeßens · Beifang Ma

Universität Duisburg-Essen, Essen, Deutschland

E-Mail: esther.winther@uni-due.de

Monika Tröster · Beate Bowien-Jansen

Deutsches Institut für Erwachsenenbildung - Leibniz-Zentrum für Lebenslanges Lernen, Bonn, Deutschland 


\title{
On the way to more collaboration: Collaborative learning as an approach in professional teacher development in literacy
}

\begin{abstract}
Collaborative professional development is an important measure to enable teachers to cope with challenges of the post-pandemic work reality. How this can be achieved or promoted in professional development in basic education/literacy has been inadequately researched so far. This article addresses this desideratum and describes exploratory the conditions for success of collaborative learning in the context of a section of collaborative professional development. Here, collaborative learning is made the subject of continuing education along a learning game validation for basic financial education. It is shown that, building a team organization, leading a team, and dividing tasks within a team are relevant to teachers' collaborative learning and that professional experience moderates the relationship between perceived efficacy of the collaboration process and the emergence of state motivation in collaboration.
\end{abstract}

Keywords Collaborative learning · Professional development · Serious game · Mixed methods · Financial literacy

\section{Modifikation von Fortbildungsangeboten für Lehrkräfte in der Grundbildung}

Die Aufgaben von Lehrkräften wandeln sich. So wird zunehmend von ihnen erwartet, dass sie (a) mit Kollegen kooperieren, (b) in Netzwerken agieren können sowie (c) individuelle Förderungen für Lernende - auch ortsunabhängig - anbieten (OECD 2020; KMK 2011). Dieses Aufgabenportfolio stellt auf die Fähigkeit zur Kollaboration als transversale Kompetenz im Professionalisierungsdiskurs ab. Kollaboration kann hierbei zwischen Lehrkräften an einer Schule oder über Bildungsorganisationen hinweg entstehen (Duncombe und Armour 2004) und generiert Vorteile, die sowohl auf individueller als auch auf institutioneller Ebene wirken: Zahlreiche Studien belegen, dass Kollaboration für die berufliche Entwicklung von Lehrkräften vorteilhaft ist (King and Newmann 2001, vgl. Literaturüberblick in Duncombe und Armour 2004) und dass sich durch kollaboratives Arbeiten und institutionelle Kooperation Ressourcenerweiterungen erzielen lassen. So können vorhandene Lernmaterialien gemeinschaftlich genutzt und kooperativ weiterentwickelt und Handlungsempfehlungen und Weiterbildungskonzepte zur professionellen Entwicklung von Lehrkräften über verschiedene Bildungssektoren hinweg eingesetzt werden (King und Newmann 2001). So gilt aus Sicht der (beruflichen) Weiterbildungsforschung u.a. die Kollaboration als eine wichtige Kompetenz für den Arbeitsmarkt (von Davier et al. 2017; Graesser et al. 2018; Hesse et al. 2015).

Die hierzu notwendige Kommunikation und Kollaboration kann über professionelle Lerngemeinschaften (Warwas et al. 2019) in besonderem Maße gefördert werden. Den institutionellen Rahmen hierfür bieten Fort- und Weiterbildungen, die Kollaboration und Kooperation als Zielkategorien integrieren. Die Ausgestaltung von Weiterbildungsinhalten und -programmen wird sich vor diesem Hintergrund zunehmend an Themen wie Teamfähigkeit und Kollaboration ausrichten (hierzu auch 
prozessorientierte Weiterbildung: Baethge und Schiersmann 1998). In diesem Zusammenhang definieren Hesse et al. (2015) folgende Merkmale von Kollaboration: Kollaboration gilt als (a) eine Kommunikation, die ein gemeinsames Verständnis schafft, (b) eine Kooperation, die zur Planung und Problemanalyse beiträgt, sowie (c) eine Responsivität, die auf eine aktive Beteiligung am Gruppenprozess zielt. Die Arbeiten von Duncombe und Armour (2004) sowie Warwas et al. (2019) beziehen sich auf den Schulbereich, können aber auf die Kollaboration von Lehrkräften im Bereich der Grundbildung übertragen werden: Die Merkmale der Kollaboration bleiben gleich (vgl. Hesse et al. 2015), während sich der (institutionelle) Anwendungskontext ändert. Vor diesem Hintergrund verfolgt der Beitrag den Anspruch, den Forschungsstand zur schulischen Lehrkräftekollaboration (hierzu u. a. Fussangel und Gräsel 2012; Kullmann 2016) mit einem Fokus auf die Grundbildung explorativ zu ergänzen.

\subsection{Lehrkräfte in der Grundbildung unter Pandemiebedingungen}

Das Fort- und Weiterbildungssystem ist auf unterschiedlichen Ebenen insbesondere durch den Ausfall aller Präsenzangebote während der Pandemie stark betroffen: Auf systemischer Ebene ist festzuhalten, dass in weiten Teilen schnell auf distante Formate umgestellt werden konnte, so dass die Angebote zwar erheblich reduziert wurden (nur ein Drittel der Weiterbildungsangebote wurden digital fortgesetzt; Flake et al. 2020, o. S.), die grundlegenden Angebotsstrukturen aber erhalten geblieben sind und auch die Teilnehmendenstrukturen keine große Veränderung erfahren haben. Mit einem Blick ins Detail wird deutlich, dass auf individueller Ebene jene Gruppe von Lernenden den Kontakt zu den Weiterbildungsangeboten verloren hat, die auch vor der Pandemie bereits unterrepräsentiert war: Menschen mit geringer formaler Bildung und Nichtberufstätige lernen deutlich seltener digital (28\%) als Erwerbstätige und Akademiker (59\%; vgl. Schmid et al. 2017, S. 7). Der Ausfall von Präsenzkursen hat damit die sozioökonomische Ungleichheit durch die bestehenden Teilnahmestrukturen in der Weiterbildung verschärft, da Formate wie Alphabetisierungskurse, Vorbereitungskurse für den Erwerb von Schulabschlüssen, Integrationskurse oder offene Lerncafés vor dem Hintergrund der pandemiebedingten Schließung von Bildungseinrichtungen nicht oder nicht in gewohnter Weise stattfinden konnten (hierzu vgl. Koppel und Langer 2020). Eine Ursache hierfür ist, dass die zur Verfügung stehenden Materialen und didaktischen Designs sehr stark auf ein Vor-Ort-Lernen und auf intensive Beziehungen zwischen Kursleitenden und Lernenden setzen und die Lehrenden trotz hohen Engagements wenig Möglichkeiten gefunden haben, die Lernenden zu erreichen und die Teilnehmendenbindung zu stärken. Mit Blick auf die in der Lehrkräftefortbildung in der Grundbildung verwendeten Lehr-Lernmaterialien zeigt sich, dass trotz eines einsetzenden Schwungs im Verlauf der Pandemie, in den aktuell stattfindenden Weiterbildungskursen für Lernende sowie für Lehrkräfte in der Grundbildung auf Unterlagen und Methoden gesetzt wird, die für ein Lehr-Lernsetting in Präsenz entwickelt wurden. Berufliches Zusammenarbeiten wird in diesen (eher traditionellen) Fortbildungsangeboten für Lehrkräfte und Weiterbildungsangebote für gering Literalisierte kaum realisiert - gerade distante Angebote können jedoch von kollaborativen Formaten in besonderer Weise profitieren: Die 
Bindung der Lehrkräfte untereinander wird gestärkt und es werden Handlungsoptionen und technische Möglichkeiten aufgezeigt, mit denen auch die Beziehungen zu den Lernenden/Kursteilnehmenden ortsunabhängig aufrechterhalten werden können.

An dieser Stelle gilt es, Lehrmaterial zu modifizieren sowie Lehrkräfte zu professionalisieren: Fortbildungen werden relevant, die Lehrkräfte in die Lage versetzen, kollaborative Lernprozesse auf Distanz bei Lernenden zu gestalten und zu unterstützen. Prozessorientierter Weiterbildung (Baethge und Schiersmann 1998) folgend ist anzunehmen, dass distante Kollaboration relevanter wird. Kollaboration sollte damit in einer kollaborativen Lernumgebung und (prozessnah) während einer distanten Kollaboration gelernt werden: Im vorliegenden Beitrag wird eine Fortbildung zum kollaborativen Lernen und Arbeiten für Lehrkräfte in der Grundbildung angeboten. Die Fortbildung zielt inhaltlich auf die Validierung eines kollaborativen Lernspiels. Die Validierung des Lernspiels erfolgt durch eine Gruppendiskussion. Inhaltlich werden v. a. das Material und der Spielprozess gemeinsam diskutiert. Die gemeinsame Validierung des Lernspiels setzt Kollaboration zwischen den Lehrkräften voraus. Pandemiebedingt werden die Inhalte des Lernspiels nicht nur kollaborativ sondern auch distant und digital von den Lehrkräften validiert. Zuletzt wird die Fortbildung theoriegeleitet evaluiert und explorativ analysiert.

\subsection{Kollaborative Lehrkräfteprofessionalisierung als Schnittstelle}

Lehrkräfteprofessionalisierung wird in der Literatur unterschiedlich definiert (Avalos 2011). Für den vorliegenden Beitrag definieren wir Lehrkräfteprofessionalisierung (Professional Development) wie folgt:

Professional Development refers to in-service training which seeks to update, develop and broaden the knowledge teachers acquired during initial education and/or provide them with new skills and professional understanding. Professional development may also be provided to accompany the implementation of educational reforms. It is distinct from further qualifying training which normally enables teachers to teach another subject or at another educational level (additional qualifications) (OECD 2005, S. 124).

Während die Weiterbildung von Lehrkräften auf den Erwerb einer zusätzlichen Lehrbefähigung, bspw. in einem weiteren Fach oder in einer anderen Jahrgangsstufe, zielt, wird die Fortbildung von Lehrkräften von Terhart (2016) als dritte Phase der Lehrkräftebildung im Anschluss an das Studium und das Referendariat definiert. Die Lehrkräfteprofessionalisierung findet entlang der Definition der OECD genau in dieser dritten Phase statt (vgl. Neuweg 2010), um theoretische Grundlagen zu aktualisieren, praktische Handlungskompetenzen zu vertiefen sowie die Qualifikation aufrechtzuerhalten. Daraus ergibt sich, dass mehr Lehrkräftefortbildungen angeboten werden sollten, um erfahrene und weniger erfahrene Lehrkräfte auf Herausforderungen und Veränderungen im Verlauf ihres beruflichen Lebens vorzubereiten und sie bei der Bewältigung zu unterstützen (OECD 2009). Im Zusammenhang mit der Pandemie gewinnt die Lehrkräftefortbildung zusätzlich an Relevanz, da durch die pandemische Situation deutlich wurde, dass Lehrkräfte kollaborativ auf Distanz arbeiten werden und dass diesen Änderungen durch Qualifizierungsmaßnahmen zu 
begegnen ist. Einerseits interagieren die Lehrkräfte mit Kolleginnen und Kollegen bspw. zur Vorbereitung von Lehr-Lern-Materialien digital und andererseits findet der Unterricht mit Lernenden online statt. Neben kooperativen Arbeitsformen, u. a. CoTeaching, werden in der aktuelleren Forschung insbesondere Professional LearningAspekte in den Blick genommen (Butler und Schnellert 2012; Friend et al. 2010; Puteh et al. 2015).

Der Forschungsstand zur Lehrkräftekooperation und -kollaboration ist eher für Lehrkräfte in schulischen Kontexten differenziert (hierzu Übersichtsartikel vgl. Fussangel und Gräsel 2014; Kullmann 2016); Befunde für Lehrkräfte in der Grundbildung fehlen. Dieses Desiderat ist umso dringlicher aufzuarbeiten, da die Fortbildung von Lehrkräften in der Grundbildung i.d.R. nicht an ein Lehramtsstudium und damit an vergleichbare curriculare Studieninhalte anknüpfen kann (vgl. Qualifikationen von (fachfremden) Fachkräften in der Grundbildung: Löffler und Korfkamp 2016). An der Schnittstelle von Lehrkräfteprofessionalisierung und Weiterbildungsforschung setzt der Beitrag einen Fokus auf die Lehrkräfteprofessionalisierung in der Grundbildung und hier im Besonderen auf die Zielsetzung, Lehrkräftekollaboration zu stärken.

Die Wichtigkeit der kollaborativen Aktivitäten wurde von zahlreichen Studien der kollaborativen Lehrkräfteprofessionalisierung (collaborative professional development; CPD) belegt (Villegas-Reimers 2003). Befunde aus dem neuesten TALISBericht (OECD 2019) bestätigen ältere Forschungsergebnisse, die auf positive Effekte der kollaborativen Lehrkräfteprofessionalisierung verweisen (hierzu DarlingHammond und McLaughlin 1995). Auch im politischen Agenda-Setting spielt die Forderung nach mehr kollaborativer Kompetenz in der Aus- und Weiterbildung von Lehrenden eine Rolle (hierzu u. a. Europäische Kommission 2010). Im Fokus dieser Forderungen stehen vor allem die Entwicklung kollaborativer Methoden sowie der Auf- und Ausbau eines kollaborativen Mindsets (u. a. Massenkeil und Rothland 2016). Forschungen zur CPD belegen, dass personenbezogene Faktoren eine erfolgreiche Kollaboration behindern können (Duncombe und Armour 2004). Lehrkräfte tendieren dazu, in ihrer gewohnten Art und Weise zu arbeiten und Veränderungen zu vermeiden, weil sie von der Notwendigkeit nicht überzeugt sind, ihren Unterricht durch neue Praktiken, z. B. Kollaboration (Duncombe und Armour 2004), zu verbessern. Guskey und Sparks (1996) argumentieren, dass erfahrene Lehrkräfte neue pädagogische Skills oder Methoden erst akzeptieren und beibehalten, wenn die Effektivität und Wirksamkeit in den Praktiken belegt werden. Diese Befunde liefern Hinweise darauf, dass die Berufserfahrung ein wichtiger personenbezogener Faktor ist und die Beziehung zwischen der Wahrnehmung der Wirksamkeit der neuen pädagogischen Skills oder Methoden und der Veränderung von Überzeugungen und Einstellungen beeinflussen kann.

Bei der Evaluation von Lehrkräftefortbildungsangeboten spielen neben den Gestaltungsmerkmalen und den kontextuellen Bedingungen auch individuelle Merkmale der Lehrkräfte eine wichtige Rolle (Fögele und Mehren 2015). Es wurde ein vierEbenen-Modell der Wirksamkeit von Lehrkräftefortbildungen entwickelt, in dem die situational-motivationalen Reaktionen der Teilnehmenden als wichtige Indikatoren berücksichtigt werden (Fögele und Mehren 2015; vgl. Tab. 1). 
Tab. 1 Ebenen der Wirksamkeit von Lehrkräftefortbildungen in Anlehnung an Fögele und Mehren (2015)

\begin{tabular}{ll}
\hline Ebene 04 & $\begin{array}{l}\text { Das Lernen der Lernenden (Performanz, Interesse etc.) } \\
\text { Ebene 03 }\end{array}$ \\
Ebene 02 & $\begin{array}{l}\text { Die Veränderungen im unterrichtspraktischen Handeln (Aufgabenkultur etc.) } \\
\text { gen etc.) }\end{array}$ \\
Ebene 01 & Die Reaktion der Teilnehmenden (Zufriedenheit, Vorbehalte etc.) \\
\hline
\end{tabular}

Während auf den beiden höheren Ebenen eine langfristige Veränderung in der Lehrpraxis angestrebt wird, zielen die unteren beiden Ebenen auf einen individuellen und situationalen Zustand, der sich nach der Intervention erfassen lässt und die Grundlage für eine dauerhafte Veränderung ist.

\section{Wirksamkeit von Lehrkräfteprofessionalisierung}

Im Fokus vieler Fort- und Weiterbildungsprogramme für Lehrkräfte steht die Verbesserung der Alltagspraxis und die Frage nach den Veränderungen in den Einstellungen, Überzeugungen und fachlichen Kompetenzen der Lehrkräfte, die dazu notwendig sind (u. a. Lewin 1935; Holt et al. 2007). Es liegen Veränderungsmodellierungen und empirische Befunde vor, die aufzeigen, dass erfahrene Lehrkräfte ihre Einstellungen und Überzeugungen gegenüber (über-)fachlichen und pädagogischen Themen erst dann ändern, wenn sie das Thema in der Praxis implementiert sehen und als wirksam erleben (Model of Teacher Change; Guskey 2002). Das Prozessmodell der Adaptation von Überzeugungen und Einstellungen ist in Abb. 1 dargestellt.

Bei der Interpretation des Modells sollen drei Anmerkungen berücksichtigt werden:

1. Das Modell impliziert, dass der Adaptationsprozess in erster Linie ein erfahrungsbasierter Lernprozess für die Lehrkräfte ist; das Erleben der Wirksamkeit gilt als Nachweis für die Notwendigkeit der (neu) erlernten Fähigkeiten und Fertigkeiten. Die Wirksamkeit kann damit vor dem Hintergrund des Veränderungsmodells als erwünschter Output nach Anwendung der neuen Fähigkeiten und Fertigkeiten verstanden werden. Nachweisbare Ergebnisse bezüglich des erwünschten Outputs sind folglich der Schlüssel für die dauerhafte Veränderung der eigenen Einstellungen und Überzeugungen.

2. Hierbei ist bemerkenswert, dass das Erleben eines Veränderungserfolges in der Alltagspraxis vor allem für erfahrene Lehrkräfte eine der zentralen Grundbedingungen für die Veränderung ihrer Einstellungen und Überzeugungen ist (vgl. Guskey 2002). Andere Studien belegen diesen Zusammenhang: In Anlehnung an den

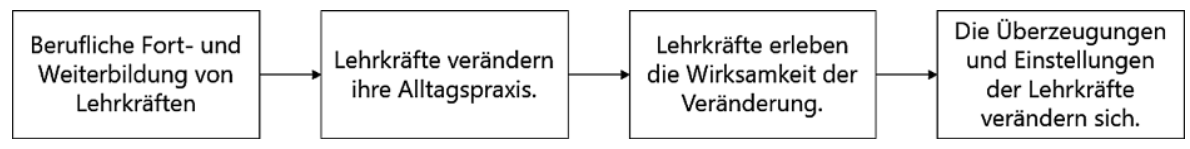

Abb. 1 Angepasstes Veränderungsmodell für Lehrkräfte in Anlehnung an Guskey (2002) 
pädagogischen Reduktionismus (Ward und O'Sullivan 1998) kann beispielsweise gezeigt werden, dass erfahrene Lehrkräfte eine bestimmte Arbeitsweise so umfassend in ihre Arbeitsroutinen implementieren, dass diese nur schwer aufzugeben ist. Erfahrene Lehrkräfte müssen demnach nachhaltig davon überzeugt werden, dass die eigene Alltagspraxis von neuen Methoden profitieren kann (Duncombe und Armour 2004).

3. Aus unterschiedlichen Arbeiten zur Persönlichkeitstheorie ist bekannt, dass sich Einstellungen und Überzeugungen (im Sinne von traits) nur langsam verändern können bzw. einen starken exogenen Anstoß benötigen, um veränderungssensitiv zu sein (vgl. hierzu Goldberg 1993; Furnham et al. 1999). Im Vorfeld der Veränderung der Überzeugungen und Einstellungen entstehen situationssensitive Motivationen (im Sinne von states). Das Zustandekommen der situationsabhängigen Motivationsfaktoren spielt auf der ersten Wirksamkeitsebene eine herausgehobene Rolle.

\section{Kollaborative Validierung eines Lernspiels}

Entlang der drei zuvor skizzierten Anmerkungen wird der Versuch unternommen, Guskeys Veränderungsmodell (2002) um Berufserfahrung und state-Motivation zu erweitern. Insofern hat die vorliegende Studie nicht nur einen evaluierenden, sondern auch explorativen Charakter. Ein gemeinsames Ziel pädagogischer Fort- und Weiterbildungen ist es, die Überzeugungen und Einstellungen von Lehrkräften zu stärken und positiv für die Gestaltung der Alltagspraxis zu nutzen (vgl. Griffin 1983). Für die Evaluation der Wirksamkeit der Fortbildungsangebote sind die Bedingungen, unter denen sich positive Veränderung der Überzeugungen einstellen, von besonderem Interesse. In der vorliegenden Studie werden daher personenbezogene Faktoren für die Veränderung der kollaborativen Einstellungen und Überzeugungen von Lehrkräften in der Fortbildung modelliert und empirisch geprüft. Hierzu wird ein Ausschnitt einer Fortbildungsmaßnahme ausgewählt und gezielt durch Inhalte angereichert, die auf Kollaborationsfähigkeit abstellen. Bei der gewählten Fortbildungsmaßnahme handelt es sich um die Implementierung von authentischen Lehr-Lernmaterialien in der Finanziellen Grundbildung (DIE 2019; Mania und Tröster 2014, 2015, 2018; PaeBens und Winther 2021; Tröster und Bowien-Jansen 2019a, b; Tröster und Schrader 2016; Winther et al. im Druck); der gewählte Ausschnitt nimmt die Validierung eines Lernspiels in den Blick. Innerhalb des zu analysierenden Ausschnitts „Lernspielvalidierung“ agieren Lehrkräfte als kollaborative Lerngemeinschaften. Damit liegt ein konstruiertes Untersuchungssetting vor, innerhalb dessen die Bedingungen und Konsequenzen des kollaborativen Lernens beobachtbar und empirisch überprüfbar werden.

\subsection{Lernspiel für die Finanzielle Grundbildung}

Der Forschungsgegenstand des vorliegenden Beitrags ist die Fortbildung „Lernspielvalidierung“ - damit ist das Lernspiel zentral. Das Lernspiel MONETTO wurde als Lernangebot für die Finanzielle Grundbildung entwickelt (vgl. hierzu Arbeiten 

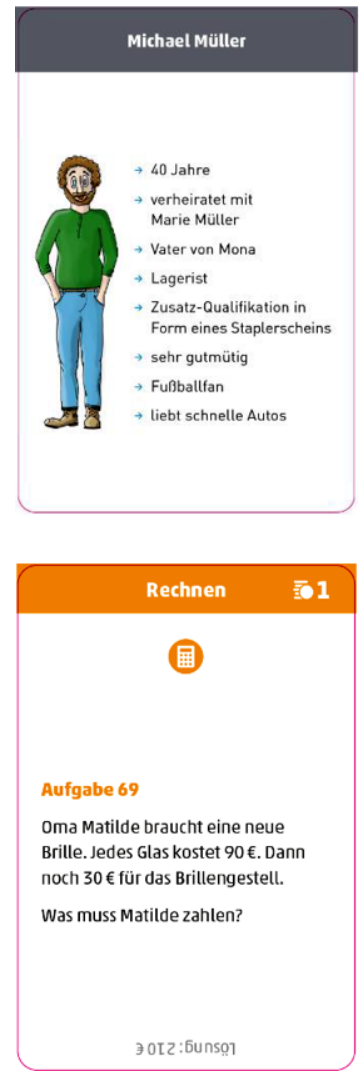
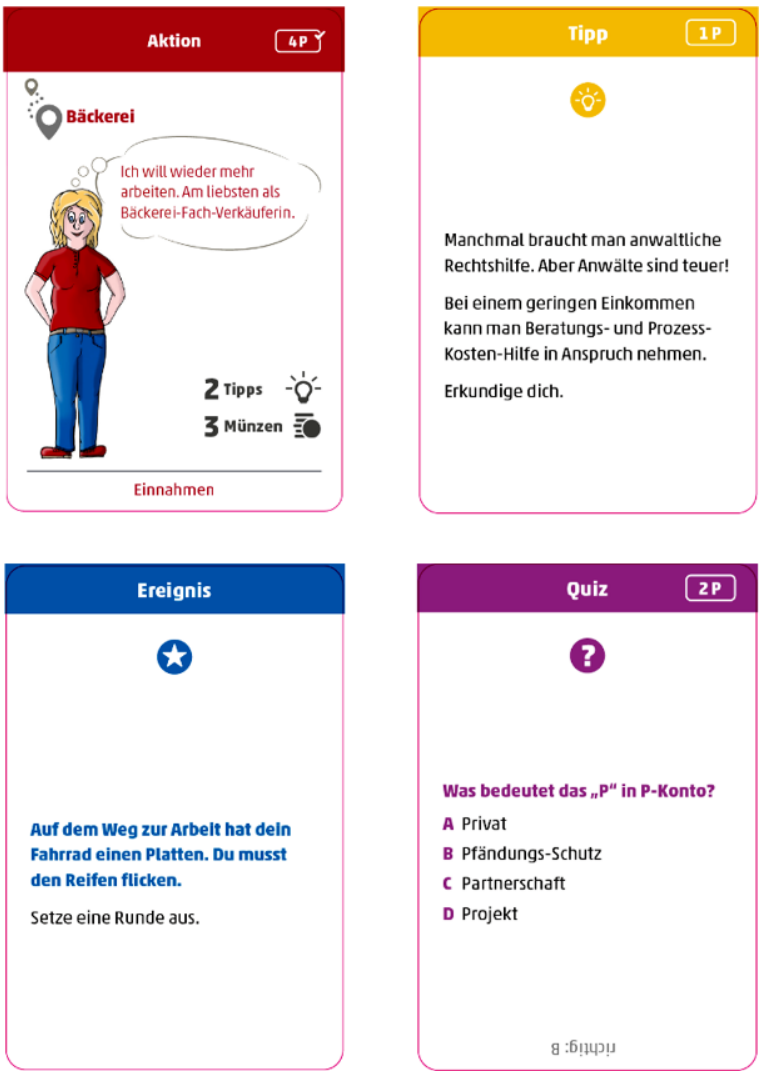

Abb. 2 Ausgewählte Spielkarten

und Materialen des Projekts CurVe'; DIE 2019; Tröster und Bowien-Jansen 2019b). Das Lernspiel für die Finanzielle Grundbildung ist ein Brettspiel. Das Spielbrett als mentaler Simulationsraum und geistiges Experimentierfeld stellt einen Stadtplan der fiktiven Stadt Maisenbohn dar (vgl. Stadtplan in Abb. 3). In Maisenbohn lebt Familie Müller (vgl. Steckbriefe in Abb. 2), die von den Spielenden bei finanziellen Herausforderungen begleitet wird. Auf einer Aktionskarte wird die Herausforderung für eine Spielfigur der Familie Müller beschrieben. Die Aktionskarte ist somit der Spielauftrag für die Spielfigur (vgl. Aktionskarte in Abb. 2). Die Geschichten auf den Aktionskarten verleihen dem Lerninhalt Sinnhaftigkeit und motivieren zum spielerischen Lernen in der Lernumgebung Maisenbohn. Da sich die Lernenden mit der Familie Müller identifizieren können, nehmen sie die Finanzgeschichten der Spielfiguren als relevant wahr, sodass die Lernaufgabe besser in die Wirklichkeit transferiert werden kann (Macleod 2008). Damit ergänzt die lernförderliche Geschichte

\footnotetext{
1 Das dieser Veröffentlichung zugrunde liegende Vorhaben wird mit Mitteln des Bundesministeriums für Bildung und Forschung unter dem Förderkennzeichen W 141300 gefördert. Die Verantwortung für den Inhalt dieser Veröffentlichung liegt bei den Autorinnen.
} 


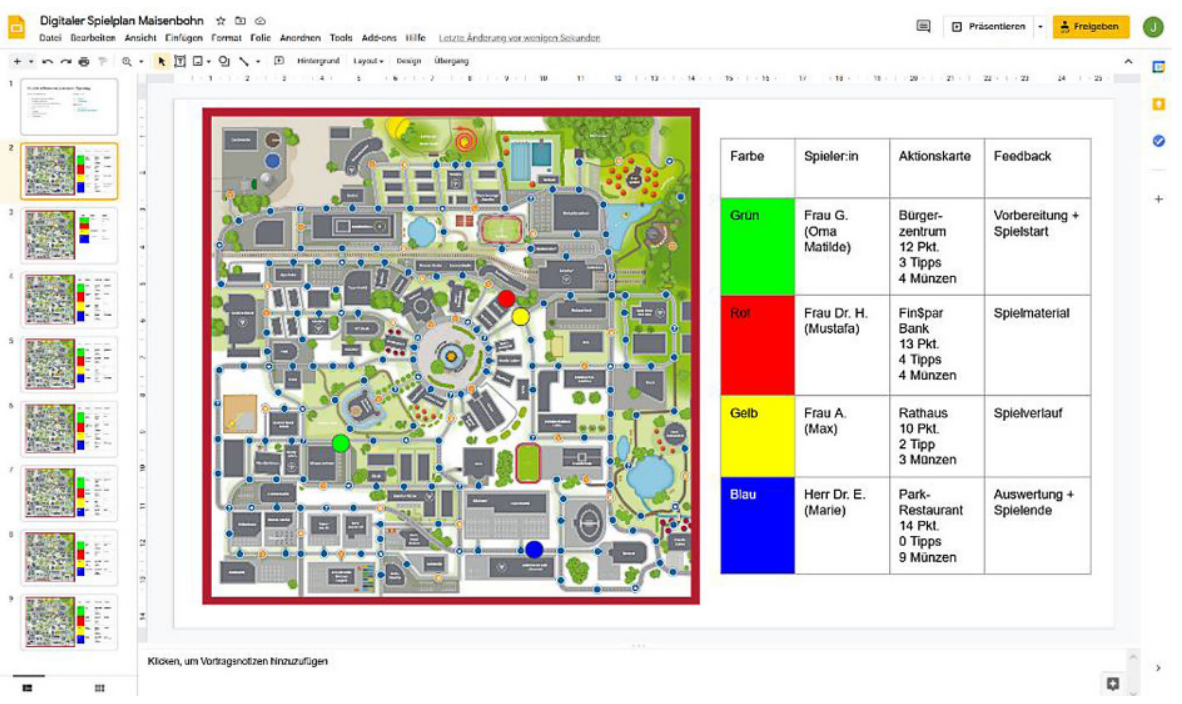

Abb. 3 Digitales Spielbrett in einer Google-Präsentation

als narrativer Anker die Authentizität, die handlungsleitend für die Instruktion ist. Das Spiels ist so angelegt, dass die Spielfiguren an einen Zielort in Maisenbohn gelangen müssen. Auf dem Weg dorthin sind von den Spielenden eine bestimmte Anzahl von Tipps und Münzen zu erspielen. Das Spiel endet, wenn eine Spielfigur das Ziel erreicht und die erforderliche Anzahl von Tipps und Münzen auf dem Weg dorthin erspielt hat. Tipps und Münzen gibt es auf entsprechend gekennzeichneten Spielfeldern, die während des Spielzugs aufgesucht werden müssen - um Münzen zu erhalten, müssen Rechenaufgaben gelöst werden (vgl. Tipp- und Rechenkarte in Abb. 2). Des Weiteren gibt es an bestimmten Orten auf dem Spielbrett Quizkarten, die zusätzliche Punkte ergeben (vgl. Quizkarte in Abb. 2). Das Quiz kann auch losgelöst vom Brettspiel allein gespielt werden. Für Überraschungen im Spielverlauf sorgen (a) Ereignisfelder auf dem Spielbrett (vgl. Ereigniskarte in Abb. 2), (b) der Hund Muffin, der Zusatzpunkte bringt, wenn Lernende eine 1 würfeln oder (c) wenn Spielende sich auf einem Feld treffen und beliebig viele Münzen und Tipps tauschen können.

Die gemeinsame Validierung des Lernspiels erfolgt auf zwei Ebenen: (1) Auf Aktionsebene wird das Spiel von den Lehrkräften kollaborativ gespielt sowie individuell und gruppenbasiert auf einer 4-stufigen Skala bewertet; (2) auf Transferebene werden die Gruppendiskussionen über das Lernspiel selbst analysiert.

Mit dem Lernspiel wird Lernmaterial zur Verfügung gestellt, das den Umgang mit Geld spielerisch vermittelt und damit niederschwellig und mit hoher Praxisrelevanz Zugang zu formalisierten Bildungsangeboten im Grundbildungsbereich ermöglicht. Das Lernspiel wurde für verschiedene Zielgruppen und Träger entwickelt und schließt sich unmittelbar an das Curriculum Finanzielle Grundbildung sowie die hierfür entwickelten Materialien an (DIE 2019). Die Quiz-, Tipp- und Rechenkarten greifen alle Subdomänen des Kompetenzmodells Finanzielle Grundbildung auf 
(vgl. Mania und Tröster 2015). Die Rechenaufgaben unterschieden sich zusätzlich zur Kompetenzdomäne auch noch in ihrer Schwierigkeit. Das Lernspiel wird nur in einer Domäne gespielt. Das Lernspiel und seine Komponenten sind sprachlich auf Alpha-Level 3 bis $4^{2}$ gestaltet, um den besonderen Bedarfen der Grundbildungskurse gerecht zu werden (Grotlüschen und Buddeberg 2020).

Die Implementierung des Lernspiels in den Kursangeboten der Alphabetisierung/ Grundbildung verfolgt eine Doppelstrategie: Es sollen (1) die individuellen literalen Kompetenzen der Kursteilnehmenden gefördert sowie (2) die Kursleitenden in ihrer Arbeit unterstützt und durch didaktische Anleitung weiter professionalisiert werden. Mit dem Lernspiel wird den Lehrkräften die Möglichkeit gegeben, alltäglich komplexe Haushalts- und Finanzfragen auf Grundbildungsniveau in einfacher Sprache und mit hohem Lebensweltbezug zu unterrichten. Während des Einsatzes des Lernspiels werden die Lehrkräfte zu Lernbegleitern, die die Kursteilnehmenden über Alltagsanforderungen an Lernprozesse heranführen und die alltägliche Relevanz der Lerninhalte aufzeigen. Das Rollenverständnis des Lernbegleiters stellt den Anker für die Fortbildungssequenz dar: Die Lehrkräfte nehmen als aktive Lernende am Spiel teil und validieren dieses prozessbegleitend in ihrer Funktion als Experten (hierzu Beck 2020). Zu Beginn des Lernspielprozesses ist es notwendig, dass die Lehrkräfte ein gemeinsames Verständnis der Regeln für ihre Spielgruppe aufbauen. Während des Spiels kann in verschiedenen Spielsituationen kollaboriert werden: Lehrkräfte können sich an der Lösung von Quizaufgaben ihrer Mitspielenden beteiligen und die einzelnen Spielstationen hinsichtlich ihrer inhaltlichen Bedeutung für den Kompetenzaufbau der Lernenden kommentieren.

Pandemiebedingt wurde das Spielbrett über eine Google-Präsentation mit allen spielenden Lehrkräften aus der Grundbildung geteilt (vgl. Abb. 3). Dies ermöglichte, die Spielzüge der einzelnen Figuren für alle Spielenden sichtbar zu machen. Ziel des Spiels ist es, die eigene Spielfigur zum Zielort zu bringen und dabei entsprechende Tipps und Münzen zu sammeln.

\subsection{Protokoll zum kollaborativen Prozess}

Das Protokoll wird geführt, um (1) Feedback zu geben und um (2) die Kollaborationsprozesse zu dokumentieren. Die Probanden geben in fünf Feedbackkategorien, die sich am Spielprozess orientieren, Bewertungen ab. Die Feedbackkategorie $1=$ Vorbereitung und Spielstart wird in Abb. 4 dargestellt (die anderen Feedbackkategorien sind: 2 = Spielmaterial; 3 = Spielverlauf; 4 = Spielauswertung und Spielende; der Aufbau der Protokolle ist identisch).

Die Lehrkräfte nutzen das Protokoll zur Beschreibung und Bewertung des Lernspiels und zur Kollaborationsbegleitung. Sie geben an, inwiefern die spezifische Feedbackkategorie für das Spiel angemessen ist und inwiefern sie das Lernspiel individuell und nach der Gruppendiskussion auf einer vierstufigen Skala empfehlen

\footnotetext{
2 Die ausgewählten Alpha-Levels beschreiben Personen, die grundlegende Schwierigkeiten beim Lesen und Schreiben auf der Ebene zusammenhängender Texte haben (Alpha-Level 3) oder beim Schreiben jenseits der Frage von Flüchtigkeitsfehlern auffällig fehlerhaft agieren (Alpha-Level 4).
} 
Abb. 4 Protokoll für die spezifische Feedbackkategorie 1: Vorbereitung und Spielstart

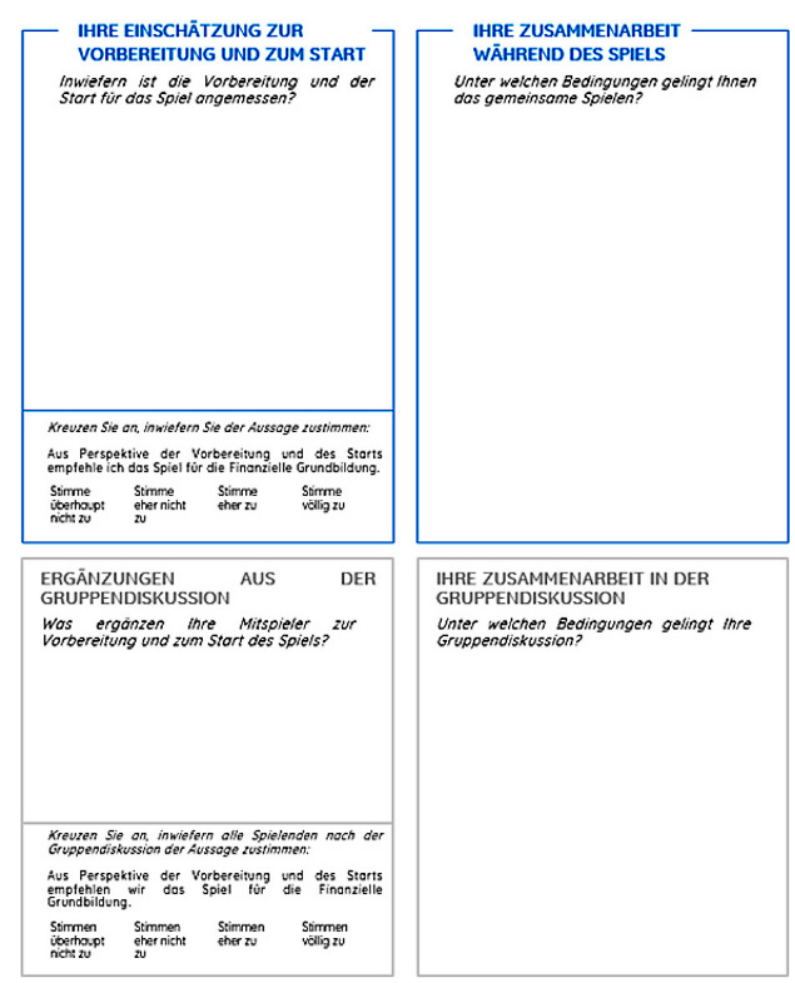

würden. Die Lehrkräfte notieren ebenfalls, unter welchen Bedingungen ihnen das gemeinsame Spielen bzw. die Gruppendiskussion gelingt.

\section{Forschungsfrage und Modellierung}

Die vorliegende Evaluationsstudie fokussiert auf personenbezogene Faktoren der Lehrkräfte, die sich in Kollaborationsprozessen als relevant erwiesen haben und deren Stärkung ein Ziel von Qualifizierungsangeboten ist: Wirksamkeitserleben sowie situationales Interesse an kollaborativer Arbeit. Im konstruierten Untersuchungssetting ,kollaborative Lernspielvalidierung“ wird explorativ erforscht, (1) wie der situationale Motivationszustand durch Fortbildungsbedingungen gefördert werden kann und (2) welche Bedingungen zum Gelingen von Kollaboration zwischen Lehrkräften in einer Fortbildung beitragen. Das Studiendesign hat einen explorativen Charakter, dass (a) neben der langfristigen Veränderung von Einstellungen und Überzeugungen in Guskeys Modell die state-Komponente der Motivation einbezieht und (b) versucht, den Einfluss von der Berufserfahrung zu erklären.

Der Analyse liegen folgende Annahmen zugrunde (Adaptation des Model of Teacher Change; Guskey 2002; vgl. Abb. 1): 
- Das Erleben von Wirksamkeit ist im Veränderungsprozess ein Startpunkt: Die Wirksamkeit der Kollaboration wird als verbaler und/oder nonverbaler Nachweis über das Ergebnis der Kollaboration interpretiert. Als Ergebnis wird eine bestehende bzw. nicht bestehende Abweichung zwischen individueller Bewertung und kollaborativer Bewertung des Lernspiels (differenziert nach Feedback-Kategorien, vgl. Abb. 4) erfasst. Weicht die eigene Bewertung einer spezifischen Feedbackkategorie von der Bewertung der Gruppe ab, erleben die Lehrkräfte den Kollaborationsprozess als nicht wirksam; gleicht das eigene Urteil dem Gruppenurteil wird dies entsprechend als Beleg für die Wirksamkeit akzeptiert.

- Das Erleben von Wirksamkeit führt zu Entstehung situationssensitiver Motivationen (im Sinne von states) der Lehrkräfte: Ein als wirksam wahrgenommener Kollaborationsprozess erhöht die Wahrscheinlichkeit, dass sich die Einstellungen und Überzeugungen der Lehrkräfte gegenüber Kollaboration positiv verändern. Als state-Komponente wird das situationale Interesse an Kollaboration erhoben, um den situationalen Motivationszustand zu beschreiben und das situationsorientierte Verhalten zu erklären.

- Die Berufserfahrung beeinflusst die Veränderungsbereitschaft auf state-Ebene (sowie auf trait-Ebene): Erfahrene Lehrkräfte halten stärker an Gewohnheiten und Routinen fest; ihre vorhandenen Einstellungen und Überzeugungen beeinflussen den Kollaborationsprozess. Mit den Skalen „Freude an der Zusammenarbeit im Team“ (folgend abgekürzt als Freude) sowie „Wertschätzung für die Zusammenarbeit im Team“ (folgend abgekürzt als Wertschätzung) werden die Einstellungen vor der Fortbildungsmaßnahme (analog zu PISA 2015 in OECD 2017) erhoben. Es wird angenommen, dass die Beziehung zwischen der wahrgenommenen Wirksamkeit des Kollaborationsprozesses und dem situationalen Interesse an Kollaboration von der Berufserfahrung mediiert wird.

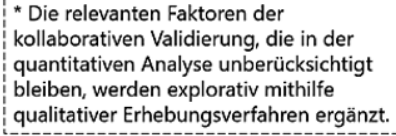

Berufserfahrung in der Erwachsenenbildung

bildung
Freude an der
Zusammenarbeit im Team
Wertschätzung für die
Zusammenarbeit im Team
Residuen (qualitativ $\mathrm{zu}$
bestimmende Faktoren)

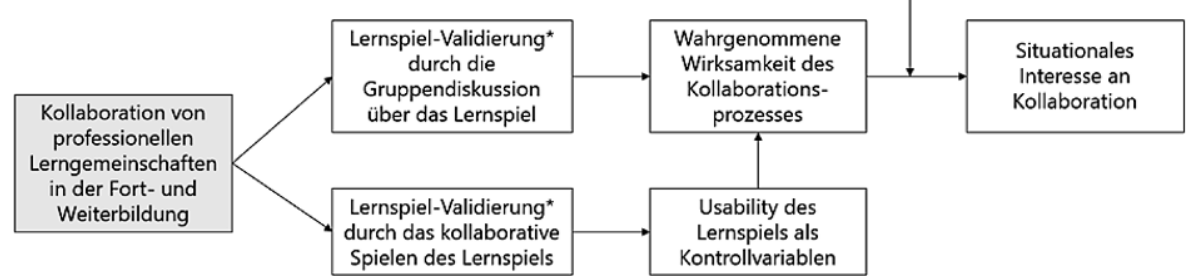

Abb. 5 Modellierung der Kollaboration von professionellen Lerngemeinschaften in Lehrkräftefortbildungen 
- Die Studie legt bewusst den Fokus auf den Prozess des kollaborativen Lernens professioneller Lerngemeinschaften; andere Gestaltungselemente des Lernspiels (hier: Usability, die sich ausdifferenziert über Basiskriterien, funktionale Kriterien, Dialogkriterien, Input-/Output-Kriterien sowie Expertenkriterien) werden kontrolliert.

Basierend auf diesen Annahmen wird die Evaluation der Fortbildung respektive die Evaluation eines Ausschnitts aus der kollaborativen Lehrkräfteprofessionalisierung entlang des folgenden Modells durchgeführt.

Die vorliegende Studie ist als Feldstudie angelegt. Dies hat zur Folge, dass bei der explorativen Analyse der Daten (a) nur begrenzt kausale Mechanismen und Korrelationen aufgedeckt werden können und (b) eine Vielzahl von Faktoren, die einen Einfluss auf gelingende Kollaboration haben könnten, in der quantitativen Analyse nicht berücksichtigt werden können (vgl. Residuen in der Abb. 5). Allerdings verspricht der Einsatz einer Feldstudie einen raschen und zumeist reliablen Überblick über die zugrundeliegenden Forschungsfragen. Um Anpassungen am Modell zu begründen und weitere Gelingensbedingungen von Kollaboration zu integrieren, wird eine explorative qualitative Teilstudie ergänzt.

\section{Methode}

Für die Evaluationsstudie wurden unterschiedlich erfahrene Lehrkräfte aus unterschiedlichen Einrichtungstypen und von unterschiedlichen Trägern aus dem gesamten Bundesgebiet für die Studie ausgewählt $(N=32)$. Von den befragten Lehrkräften, die ihr Geschlecht angegeben haben, sind 19 weiblich und 10 männlich, die Berufserfahrung liegt durchschnittlich bei acht Jahren ( $\mathrm{SD}=7,53$ Jahre, Min= $1 \mathrm{Jahr}$, Max = 28 Jahre). Die Daten wurden im April 2021 im Rahmen eines bundesweiten Lernspieltages bei acht Spielgruppen erhoben. Jede Gruppe besteht aus vier Lehrkräften. Die Evaluationsumgebung besteht aus dem Lernspiel, einem Protokoll (vgl. Abschn. 3.2) und einem Fragebogen.

Im Fragebogen werden die folgenden Skalen erhoben:

- Usability des Lernspiels unter Berücksichtigung von Basiskriterien, funktionalen Kriterien, Dialogkriterien, Input-/Output-Kriterien sowie Expertenkriterien (vgl. Sangmeister et al. 2018; Beck 2020)

- Situationales Interesse an Kollaboration (in Anlehnung an PISA 2015; OECD 2017)

- Vorhandene Einstellungen zur Zusammenarbeit im Team über Freude und Wertschätzung (analog zu PISA 2015; OECD 2017)

- Soziodemografie (u.a. Berufserfahrung)

Während der Erhebung wurden die Lehrkräfte gebeten, einen Prototyp des Lernspiels in 120 min kollaborativ zu validieren. Der Ablauf ist in Tab. 2 dargestellt.

Jede Lehrkraft erhält einen individuellen Feedback-Auftrag. Im Anschluss an das Spielen des Lernspiels halten die Lehrkräfte ihre individuellen Rückmeldungen (einschließlich ihrer Empfehlungen für die ihnen zugewiesene Feedback-Kategorie) 
Tab. 2 Ablauf der Erhebung

\begin{tabular}{ll}
\hline Dauer & Kollaborative Validierung eines Lernspiels \\
\hline $50 \mathrm{~min}$ & Gemeinsames Spielen \\
$10 \mathrm{~min}$ & Beendigung des Spiels und Auswertung des aktuellen Spielstands \\
$10 \mathrm{~min}$ & $\begin{array}{l}\text { Notation von (a) Rückmeldung, (b) Individualempfehlung für eine spezifische } \\
\text { Feedbackkategorie }{ }^{\mathrm{a}} \text { und (c) Gelingensbedingungen für das gemeinsame Spielen }\end{array}$ \\
$20 \mathrm{~min}$ & Gruppendiskussion aller Feedbackkategorien \\
$10 \mathrm{~min}$ & $\begin{array}{l}\text { Notation von (a) Ergänzungen aus der Gruppendiskussion und (b) Gruppenempfehlung } \\
\text { sowie (c) Gelingensbedingungen für die Gruppendiskussion }\end{array}$ \\
$20 \mathrm{~min}$ & Bearbeitung des Fragebogens \\
\hline
\end{tabular}

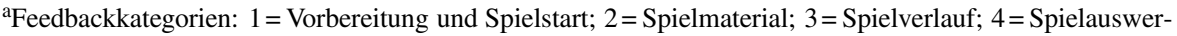
tung und Spielende

sowie die Gelingensbedingungen des gemeinsamen Spielens mittels des standardisierten Protokolls fest. In einer anschließenden 20-minütigen halbstandardisierten Gruppendiskussion werden die einzelnen Rückmeldungen präsentiert und gegeneinander abgeglichen. Abschließend notieren die Lehrkräfte Ergänzungen in ihren spezifischen Feedbackkategorien und geben die Gruppenempfehlung in der spezifischen Feedback-Kategorie an (4-stufige Likertskala von $0=$ stimmen überhaupt nicht zu bis $3=$ stimmen völlig zu: ,Aus Perspektive [z.B. der Vorbereitung und des Spielstarts] empfehlen wir das Spiel für die Finanzielle Grundbildung"). Bevor die Lehrkräfte den Fragebogen ausfüllen, protokollieren sie, unter welchen Bedingungen ihre Gruppendiskussion gelingt.

\section{Ergebnisse}

Bevor sich Gelingensbedingungen von Kollaboration in professionellen Lerngemeinschaften empirisch ableiten lassen, wird der Ausschnitt der kollaborativen Lehrkräfteprofessionalisierung entlang des zugrundeliegenden Wirkungsmodells geprüft. Aufgrund der Komplexität des Modells wird die Überprüfung in zwei Schritte unterteilt:

- Im ersten Schritt wird überprüft, ob die Berufserfahrung als Moderator die Beziehung zwischen wahrgenommener Wirksamkeit des Kollaborationsprozesses und situationalem Interesse an Kollaboration beeinflusst (vgl. Abb. 1). Die Identifikation dieses Moderatoreffekts ist unter Rückgriff auf das Teacher Change Model (Guskey 2002) bedeutsam, da angenommen wird, dass erfahrene Lehrkräfte eine Veränderung ihrer Einstellungen und Überzeugungen an das Erleben der Wirksamkeit binden.

- Im zweiten Schritt werden mediierende Faktoren in das Modell integriert, um Antworten darauf geben zu können, warum die Berufserfahrung das Zustandekommen der state-Komponente der Motivation beeinflusst und wie die vorhandenen Einstellungen und Überzeugungen, die die Lehrkräfte im Laufe ihrer Berufserfahrung entwickelt haben, den Kollaborationsprozess beeinflussen. Es wird angenommen, dass die Einstellungen und Überzeugungen der Lehrkräfte (hier über die Skalen 
„Freude an der Zusammenarbeit in Team“ sowie „Wertschätzung für die Zusammenarbeit im Team“ operationalisiert; vgl. Abb. 1) den Zusammenhang zwischen der Berufserfahrung der Lehrkräfte und dem situationalen Interesse an Kollaboration mediieren.

Auf Basis dieser Analyseschritte wird die Beziehung zwischen der wahrgenommenen Wirksamkeit des Kollaborationsprozesses für die eigene Arbeit, der Entstehung des situationalen Interesses an Kollaboration und der Berufserfahrung der Lehrkräfte aufgeklärt. Um darüber hinaus Aussagen treffen zu können, welche Gelingensbedingungen sich für erfolgreiche Kollaborationsprozesse in der Lehrkräfteprofessionalisierung identifizieren lassen, werden die qualitativen Daten des Protokolls explorativ ausgewertet.

\subsection{Empirische Modellprüfung}

Einen deskriptiven Überblick aller Mess- und Skalenkennwerte liefert Tab. 3. Mittels Kruskal-Wallis-Test wurde geklärt, ob sich Unterschiede bezüglich der Ausprägungen von Berufserfahrungen zwischen den Spielgruppen zeigen. Mit Blick auf die vorliegenden Daten kann ein derartiger Unterschied nicht gesichert werden $(\mathrm{H}(7)=12,079, p=0,060)$. Es lässt sich zunächst feststellen, dass das situationale Interesse an der kollaborativen Validierung des Lernspiels hoch ausgeprägt ist $(\mathrm{M}=3,33, \mathrm{SD}=0,62$ auf der 4-stufigen Skala des situationalen Interesse; vgl. Tab. 3).

Das Modell kollaborativer Lerngemeinschaften (vgl. Abb. 5) wird mithilfe von PROCESS SPSS (Hayes 2018) validiert. Die Moderationsanalyse des ersten Schritts wird in Tab. 4 dargestellt. Die Regressionsanalyse, in der alle fünf Dimensionen der

Tab. 3 Skalenkennwerte wichtiger Indikatoren

\begin{tabular}{|c|c|c|c|c|c|}
\hline Indizes & Min & Max & M & SD & $\begin{array}{l}\text { Cronbachs } \\
\text { Alpha }\end{array}$ \\
\hline Berufserfahrung in Jahren (1 Item) & 1 & 28 & 8,67 & 7,53 & - \\
\hline $\begin{array}{l}\text { Freude an der Zusammenarbeit im Team } \\
\text { (4 Items) }\end{array}$ & 2 & 4 & 3,50 & 0,56 & 0,888 \\
\hline $\begin{array}{l}\text { Wertschätzung für die Zusammenarbeit } \\
\text { im Team (4 Items) }\end{array}$ & 1,75 & 4 & 3,09 & 0,63 & 0,738 \\
\hline Lernspiel: Basiskriterien (3 Items) & 1 & 4 & 2,79 & 0,63 & 0,911 \\
\hline $\begin{array}{l}\text { Lernspiel: Funktionale Kriterien } \\
\text { (3 Items) }\end{array}$ & 1 & 4 & 2,61 & 0,57 & 0,797 \\
\hline Lernspiel: Dialogkriterien (11 Items) & 1 & 4 & 3,09 & 0,56 & 0,914 \\
\hline $\begin{array}{l}\text { Lernspiel: Input-/Outputkriterien } \\
\text { (4 Items) }\end{array}$ & 1 & 4 & 3,14 & 0,67 & 0,850 \\
\hline Lernspiel: Expertenkriterien (6 Items) & 1 & 4 & 2,92 & 0,60 & 0,660 \\
\hline Situationales Interesse (3 Items) & 2 & 4 & 3,33 & 0,62 & 0,935 \\
\hline $\begin{array}{l}\text { Wahrgenomme Wirksamkeit des Kolla- } \\
\text { borationsprozesses }\end{array}$ & 0 & 3 & 0,67 & 0,90 & - \\
\hline
\end{tabular}

Die Abweichung der individuellen Empfehlung von der Gruppenempfehlung wurden als Absolutwert ausgewertet. Wenn die Abweichung groß ist, erlebt die Lehrkraft dies eher als Misserfolg. Während eine kleine Abweichung eher als Erfolg wahrgenommen wird 
Tab. 4 Ergebnis der Moderationsanalyse mit situationalem Interesse als Kriterium

\begin{tabular}{llllll}
\hline & Prädiktoren & Koeffizient & SE & t & $P$ \\
\hline Konstant & & 2,552 & 0,553 & 4,618 & 0,000 \\
Wahrgenommene & & 0,389 & 0,262 & 1,484 & 0,152 \\
Wirksamkeit & & & & \\
Berufserfahrung & & 0,036 & 0,017 & 2,147 & 0,043 \\
Interaktionsterm & & $-0,511$ & 0,019 & $-2,655$ & 0,014 \\
Kontrollvariablen & Basiskriterien & 0,100 & 0,294 & 0,340 & 0,737 \\
& Funktionale Kriterien & $-0,307$ & 0,300 & $-1,025$ & 0,316 \\
& Dialogkriterien & 0,417 & 0,372 & 1,123 & 0,273 \\
& Input-/Outputkriterien & $-0,035$ & 0,242 & $-0,145$ & 0,886 \\
& Expertenkriterien & $-0,166$ & 0,296 & $-0,056$ & 0,956 \\
\hline
\end{tabular}

Tab. 5 PROCESS-Output der Mediationsanalyse mit situationalem Interesse als Kriterium

\begin{tabular}{llllll}
\hline & Prädiktoren & Koeffizient & SE & t & $P$ \\
\hline Konstant & & 0,214 & 0,683 & 0,313 & 0,757 \\
Berufserfahrung & & 0,010 & 0,012 & 0,885 & 0,385 \\
$\begin{array}{l}\text { Freude an der Zusam- } \\
\text { menarbeit im Team }\end{array}$ & & 0,735 & 0,186 & 3,950 & 0,001 \\
$\begin{array}{l}\text { Wertschätzung für die } \\
\text { Zusammenarbeit im }\end{array}$ & & & & \\
Team & & $-0,041$ & 0,154 & $-0,263$ & 0,800 \\
Kontrollvariablen & Basiskriterien & 0,140 & & & \\
& Funktionale Kriterien & $-0,444$ & 0,268 & 0,523 & 0,606 \\
& Dialogkriterien & 0,384 & 0,338 & $-1,650$ & 0,113 \\
& Input-/Output-Kriterien & $-0,129$ & 0,222 & $-0,579$ & 0,269 \\
& Expertenkriterien & 0,197 & 0,280 & 0,704 & 0,489 \\
\hline
\end{tabular}

Usability des Lernspiels kontrolliert werden, zeigt, dass das Gesamtmodell signifikant ist $\left(\mathrm{R}^{2}=71,02 \%, \mathrm{~F}(8,23)=2,93, p=0,02\right)$. Die Regressionskoeffizienten der Berufserfahrung $(\mathrm{t}=2,147, p=0,043)$ und der Interaktionseffekt von wahrgenommener Wirksamkeit des Kollaborationsprozesses und Berufserfahrung $(\mathrm{t}=-2,66, p=0,014)$ sind signifikant. Steigt die Berufserfahrung um eine Einheit, nimmt das situationale Interesse an Kollaboration um 0,036 Einheiten ab. Die wahrgenommene Wirksamkeit des Kollaborationsprozesses wirkt sich je nach Berufserfahrung unterschiedlich auf das situationale Interesse an Kollaboration aus. Der Moderatoreffekt der Berufserfahrung ist damit identifiziert.

Im zweiten Schritt wird - mittels einer PROCESS-Mediationsanalyse - überprüft, ob die vorhandenen Einstellungen und Überzeugungen der Lehrkräfte zu Kollaboration die Beziehung zwischen der Berufserfahrung und dem situationalen Interesse an Kollaboration mediieren. Die Mediationsanalyse nimmt zur Identifizierung des Mediatoreffekts die Freude und Wertschätzung an der Zusammenarbeit im Team als Mediatoren zwischen Berufserfahrung und situationalem Interesse an Kollaboration auf. Auch bei der Mediationsanalyse werden die fünf Indikatoren der Usability des Lernspiels kontrolliert. Das Mediationsmodell erweist sich nicht als signifikant (vgl. Tab. 5). 


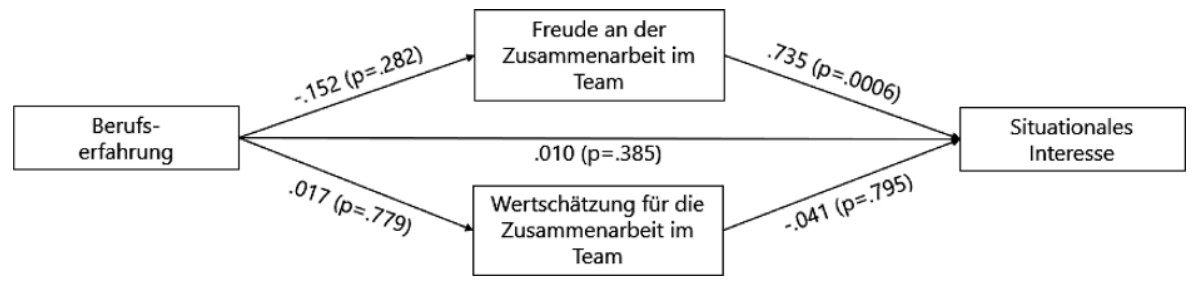

Abb. 6 Ergebnis der Mediationsanalyse mit Koeffizienten und $p$-Value

Die Ergebnisse der Mediationsanalyse (visualisiert in Abb. 6) verweisen darauf, dass die Freude an der Zusammenarbeit im Team das situationale Interesse an Kollaboration signifikant im Kollaborationsprozess beeinflusst; der Mediationseffekt der vorhandenen Einstellungen und Überzeugungen auf die Beziehung von Berufserfahrung und situationalem Interesse an der Kollaboration lässt sich hingegen nicht identifizieren.

Die über die Moderations- und Mediationsanalyse generierten Ergebnisse lassen sich wie folgt zusammenfassen: In der kollaborativen Lehrkräfteprofessionalisierung hängt die Entstehung des situationalen Interesses an Kollaboration nicht allein von dem Erleben der Wirksamkeit des Kollaborationsprozesses ab. Vielmehr beeinflusst die Berufserfahrung die Beziehung zwischen der wahrgenommenen Wirksamkeit des Kollaborationsprozesses und der Veränderung des situationalen Interesses an Kollaboration (Moderatoreffekt). Der Einfluss der Berufserfahrung wird jedoch nicht durch die vorhandenen Einstellungen und Überzeugungen zur Kollaboration mediiert.

\subsection{Gelingensbedingungen für Kollaboration in professionellen Lerngemeinschaften}

Über das Protokoll werden qualitative Daten erzeugt, aus denen sich Gelingensbedingungen von Kollaboration identifizieren lassen: Die Lehrkräfte haben über standardisierte Fragen Auskünfte zu den Bedingungen für (a) ein gelingendes gemeinsames Spielen sowie (b) zu gelingenden Gruppendiskussionen gegeben. Das Textmaterial wurde inhaltsanalytisch ausgewertet (Rädiker und Kuckartz 2019; Mayring 2015). Hierbei wurden Schwerpunktkategorien identifiziert und die einzelnen Aussagen entsprechend klassifiziert, in dem sie verschlagwortet und kategorisiert wurden. Auf Basis dieses Vorgehens konnten sieben Gelingensbedingungen identifiziert werden. Alle Kategorisierungen erfolgten von zwei Experten unabhängig voneinander. In der formativen Reliabilitätsprüfung wurden zehn zufällige Fragebögen herangezogen und durch zwei Auswertende analysiert. Es fanden sich insgesamt 20 Einträge, die kodiert wurden. Krippendorff's Alpha von 0,78 kann als akzeptabel beurteilt werden. Die Abweichungen wurden in einer Diskussion besprochen, sodass ein gemeinsamer Konsens erzielt wurde. Nach dieser Korrektur des Kategoriensystems wurde die summative Intercoderreliabilität berechnet; Krippendorff's Alpha erhöht sich dadurch auf 0,90 und kann als hoch reliabel angesehen werden. 


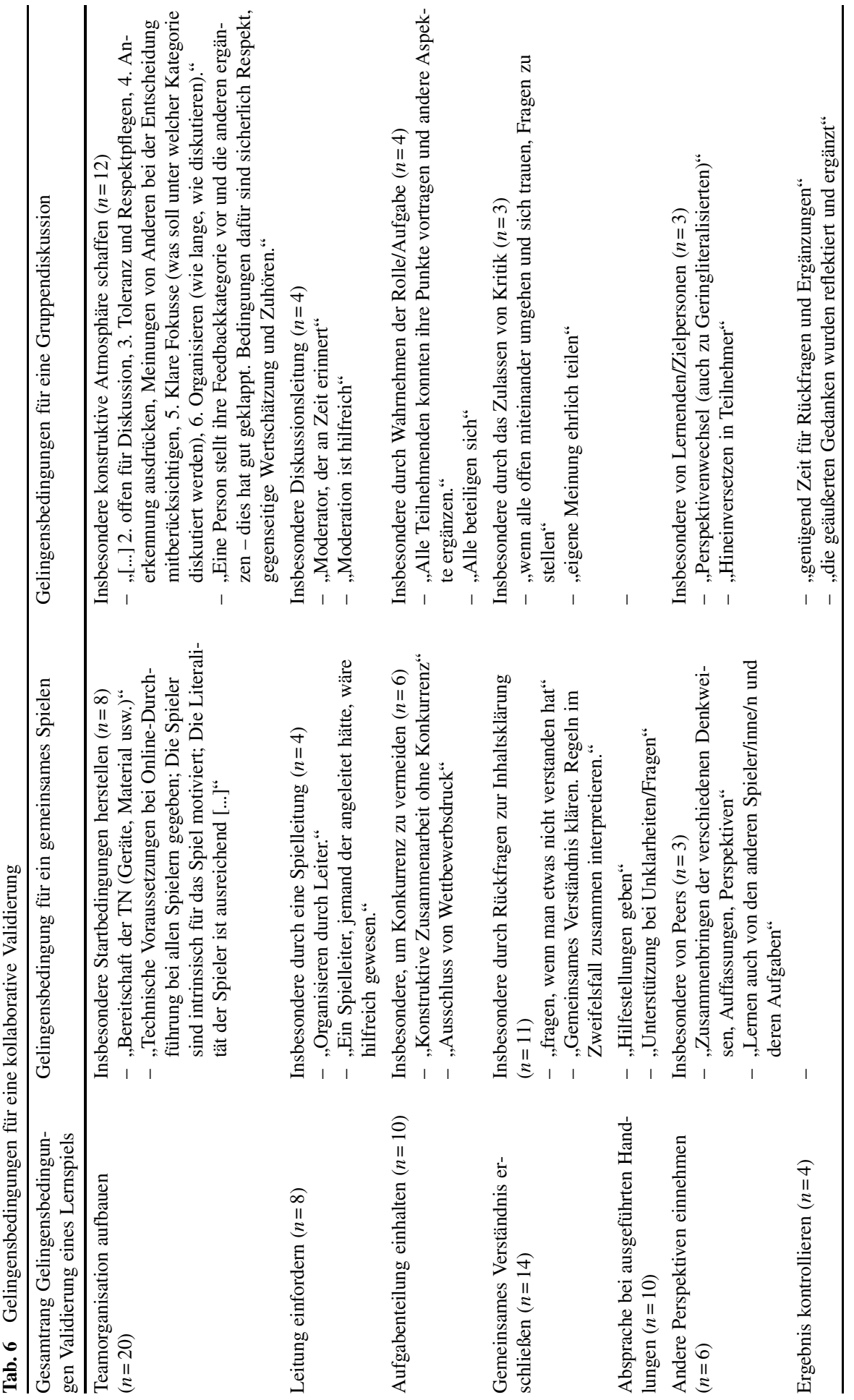


In Tab. 6 werden die identifizierten Gelingensbedingungen für eine kollaborative Zusammenarbeit professioneller Lerngemeinschaften (hier exemplarisch am Beispiel der kollaborativen Lernspielvalidierung) präsentiert. Folgende Ergebnisse lassen sich zusammenfassend beschreiben:

- Für die kollaborative Zusammenarbeit ist es notwendig, eine Teamorganisation aufzubauen. Die Teamorganisation ist davon geprägt, dass vorab die technischen Voraussetzungen für die Zusammenarbeit geklärt werden und alle Mitglieder Zugriff auf die geteilten Dokumente bzw. Informationen haben. Sie kann von einer Vergabe von Rollen und einer klaren Arbeitsteilung bzw. Aufgabenzuschreibung sowie einer kollegialen, vertrauensvollen Arbeitsatmosphäre profitieren.

- Auch kollaborative Zusammenarbeit setzt Leitung voraus. Innerhalb einer kollaborativen Lerngemeinschaft wird eine Person benötigt, die das Spiel anleitet sowie die Diskussion moderiert. Auch in diesem Zusammenhang ist die Vergabe von Rollen für die Steuerung des Kollaborationsprozesses unterstützend.

- Kollaborative Zusammenarbeit basiert auf Aufgabenteilung. Die Aufgabenteilung stellt sicher, dass die individuellen Beiträge zur kollaborativen Zusammenarbeit - auch ohne Konkurrenzkampf - geleistet werden können.

- Kollaborative Zusammenarbeit setzt ein gemeinsames, kontextuelles Verständnis innerhalb der kollaborativen Lerngemeinschaft voraus. Dieses Verständnis wird kollaborativ erschlossen. Die Lehrkräfte stellen untereinander verständnisleitende Fragen und können ergebnisoffen diskutieren.

- Bei einer gelingenden kollaborativen Zusammenarbeit unterstützen sich die Teammitglieder gegenseitig bei geplanten Handlungen, die sich auf das gemeinsame Ziel richten.

- Kollaborative Zusammenarbeit entwickelt sich durch Perspektivübernahme. Hierbei spielen die Perspektiven von Kolleginnen und Kollegen eine vergleichbare Rolle, wie die Perspektiven der Lernenden/Kursteilnehmenden.

- Kollaborative Zusammenarbeit wird durch eine gemeinsame Ergebniskontrolle gestärkt. Die kollaborativen Lerngemeinschaften begleiten den Prozess formativ und kontrollieren die jeweils aktuellen Ergebnisse durch Rückfragen und Ergänzungen. Die gemeinsame Ergebniskontrolle schließt auch eine Redefinition der Rollenzuschreibungen ein.

Die Befunde der qualitativen Analyse sind anschlussfähig. So wird Kollaboration im Kontext der PISA-Studien über drei Dimensionen definiert: (1) Gemeinsames Verständnis schaffen und aufrechterhalten, (2) angemessen Handeln, um das Problem zu lösen und (3) Teamorganisation aufbauen und aufrechterhalten (OECD 2017; Zehner et al. 2019). Diese drei Dimensionen können für die Zusammenarbeit in kollaborativen Lerngemeinschaften als hoch wirksam eingeschätzt werden; mit Blick auf kollaborative Lerngemeinschaften von Lehrkräften scheinen Gelingensbedingungen hinzuzukommen, die die pädagogischen Aufgaben und instruktionalen Herausforderungen in besonderer Weise betonen. Hierzu zählen insbesondere die Fähigkeiten zur Perspektivübernahme und das Einhalten von Arbeitsprozessschritten und Aufgabenzuschreibungen. Auch wird deutlich, dass die Berufserfahrung als Moderator eine Rolle spielt: Lehrkräfte mit höherer Berufserfahrung haben beispielsweise Teamorganisationen häufiger aktiv mitgestaltet. Vor dem Hintergrund 
ihrer individuellen Erfahrungen fordern erfahrene Lehrkräfte für die Gestaltung des Kollaborationsprozesses deutlicher eine Führung bzw. Leitung ein als Novizen; sie beharren stärker auf den ihnen bekannten Problemlösestrategien und -methoden und stellen das kollaborativ entwickelte, gemeinsame Verständnis umfangreicher zur Diskussion als ihre weniger erfahrenen Kollegen.

\section{Diskussion}

Der Beitrag hat kollaborative, professionelle Lerngemeinschaften in der Grundbildung in den Blick genommen. Hierfür wurde im Rahmen einer Fortbildungsmaßnahme ein inhaltlicher Aspekt - hier eine Lernspielvalidierung - explorativ analysiert und durch Elemente kollaborativer Zusammenarbeit angereichert. Auf diesem Weg konnte im konstruierten Ausschnitt der Lehrkräfteprofessionalisierung Kollaboration beobachtet und empirisch erfasst werden. Theoriegeleitet wurde das Weiterbildungsangebot bezüglich der Kollaboration evaluiert. Als übergeordnete Ziele von Fortbildungsangeboten gelten die positive Veränderung der Überzeugungen und Einstellungen der Lehrkräfte. Unter Rückgriff auf das Model of Teacher Change (Guskey 2002) ließen sich die Bedingungen dieser Veränderungen theoretisch identifizieren. Als Voraussetzung der dauerhaften Veränderung der Überzeugungen wurden in diesem Beitrag neben (a) der wahrgenommenen Wirksamkeit der neuen pädagogischen Fähigkeiten und Fertigkeiten sowie (b) der Berufserfahrung auch (c) die Entstehung der situationalen Motivation berücksichtigt. Bei der Analyse werden alle diese drei Aspekte berücksichtigt, um zu prüfen, ob die theoretischen Bedingungen einer dauerhaften Veränderung in Einstellungen und Überzeugungen erfüllt sind.

Die Befunde bestätigen, dass die Berufserfahrung zwischen dem Erleben von Wirksamkeit neu gelernter pädagogischer Skills bzw. Methoden und der Entstehung der situationalen Motivation vermittelt: Gerade Einstellungen und Überzeugungen erfahrener Lehrkräfte gegenüber neuen (über-)fachlichen und pädagogischen Themen einer Fort- und Weiterbildung verändern sich erst dann, wenn ihre Wirksamkeit in der instruktionalen Praxis erfahrbar ist (Guskey 2002). Vor dem Hintergrund dieser theoretischen Annahme wurde in der vorliegenden Studie insbesondere auf die moderierte Beziehung zwischen wahrgenommener Wirksamkeit und situationalem Interesse an der Kollaboration durch die Berufserfahrung fokussiert. Die Befunde zeigen, dass das entsprechende Moderationsmodell signifikant ist - die wahrgenommene Wirksamkeit des Kollaborationsprozesses wirkt sich je nach Berufserfahrung unterschiedlich auf das situationale Interesse an Kollaboration aus. Die Berufserfahrung ist, wie in den grundlegenden theoretischen Annahmen formuliert, ein zentraler Faktor, der die Beziehung zwischen der wahrgenommenen Wirksamkeit einer kollaborativen Lehrkräfteprofessionalisierung und den Motivationen der Lehrkräfte (hier zunächst auf state-Ebene) beeinflusst.

Um explorativ zu beantworten, welche Komponenten der Berufserfahrung entscheidend dafür sind, die Beziehung zwischen der wahrgenommenen Wirksamkeit neu gelernter pädagogischer Skills bzw. Methoden und die Veränderung der Einstellungen und Überzeugungen zu mediieren, wurde angenommen, dass die vor der Fortbildung vorhandenen Einstellungen und Überzeugungen der erfahrenen Lehrkräfte 
manifest sind; die Beziehung zwischen Berufserfahrung und der Veränderung des situationalen Interesses an Kollaboration sollte daher von spezifischen Einstellungen zur Kollaboration (hier Freude an der Zusammenarbeit in Team und Wertschätzung der Zusammenarbeit im Team) mediiert werden. Dieser Mediationseffekt lässt sich anhand der vorliegenden Daten nicht empirisch sichern. Welchen Einfluss vorhandene Einstellungen und Überzeugungen auf den Kollaborationsprozess haben, ist damit in weiteren Studien aufzuklären.

Unter Rückgriff auf die Befunde der quantitativen Analyse lässt sich insgesamt feststellen, dass der Ausschnitt „Lernspielvalidierung“ der kollaborativen Lehrkräfteprofessionalisierung das situationale Interesse gut adressiert (vgl. deskriptive Statistik in Tab. 3). Entlang des Evaluationsmodells der Wirksamkeit von Fortbildungsangeboten (vgl. Tab. 1; Fögele und Mehren 2015) kann dieser Ausschnitt auf Ebene 01 und teilweise auf Ebene 02 positiv bewertet werden. Die positive Beziehung zwischen der Entstehung situationaler Interessen und dem Wirksamkeitserleben im Kollaborationsprozess hängt u. a. von der Berufserfahrung ab. Die dahinterstehenden mediierenden Mechanismen wurden mit dem vorliegenden Forschungsdesign (noch) nicht aufgedeckt.

Basierend auf den quantitativen Befunden lässt sich zusammenfassen, dass beim Design der kollaborativen Fortbildungsangebote für Lehrkräfte folgende Aspekte berücksichtigt werden sollten:

1. Direktes Feedback: Das Feedback bietet den Lehrkräften die Möglichkeit, die Wirksamkeit der neu gelernten pädagogischen Skills bzw. Methoden wahrzunehmen. Das Geben von Feedback in einem digitalen und kollaborativen Lernsetting kann durch verschiedene Techniken (z.B. Audience Response Systeme) unterstützt werden (vgl. Hunsu et al. 2016). Es kann verbal oder nonverbal sein.

2. Förderung von state-Komponenten der Motivation: Während der Lerneinheit der Fortbildung sollte die state-Motivation von Lehrkräften gefördert werden. Eine dauerhafte Veränderung der Überzeugungen und Einstellungen ist das Ziel. Der langfristige Prozess setzt jedoch das Zustandekommen der state-Motivation voraus.

3. Berufserfahrung von Lehrkräften: Die Wirkung der subjektiv wahrgenommenen Wirksamkeit der neu gelernten pädagogischen Skills bzw. Methoden auf die Erhöhung der state-Motivation wird von der Berufserfahrung mediiert. Für eine Einheit der Erhöhung der state-Motivation brauchen die erfahrenen Lehrkräfte ein intensiveres Erleben der Wirksamkeit von neu gelernten pädagogischen Skills bzw. Methoden.

4. Lerngelegenheiten nach der Fortbildung anbieten: Arbeitsplatzintegrierte Followup-Maßnahmen sind anzubieten, um Veränderungen der Überzeugungen und Einstellungen der Lehrkräfte langfristig zu fördern und zu sichern. Erst durch die Implementierung kann die Fortbildung ihre langfristigen Ziele erreichen.

Darüber hinaus konnten Gelingensbedingungen für erfolgreiche Kollaborationsprozesse aus einer qualitativen Inhaltsanalyse abgeleitet werden. Die so identifizierten Faktoren sind in hohem Maße an die Literatur zum kollaborativen Lernen und zu kollaborativen, professionellen Lerngemeinschaften anschlussfähig. Um das kollaborative Lernen in Bildungsorganisationen einzuführen, sollten zehn Merkma- 
le von erfolgreicher Kollaboration umgesetzt werden (Darling-Hammond 1994, zit.

n. Duncombe und Armour 2004):

- wechselseitiges Interesse und gemeinsame Ziele

- gegenseitiges Vertrauen und Respekt

- gemeinsame Entscheidungsprozesse

- klarer Fokus

- überschaubare Lehrinhalte

- Engagement der Leitung

- steuerliche Unterstützung

- langfristiges Commitment

- dynamische Natur

- Informationsaustausch und Kommunikation

Die Befunde der qualitativen Analyse sind für zukünftige Untersuchungen nutzbar; die identifizierten Gelingensbedingungen können als Mediatoren indirekt Einfluss auf die Beziehung zwischen Berufserfahrung und Einstellungen und Überzeugungen der Lehrkräfte nehmen, da sie die Wahrscheinlichkeit erhöhen, die Wirksamkeit von Kollaboration in der eigenen, individuellen Praxis wahrzunehmen und zu erleben. Hier weitere Forschung anzuschließen, verspricht einen hohen praktischen Nutzen, da sich Empfehlungen herausarbeiten lassen, wie die Einführung kollaborativen Lernens in die bestehenden Organisationsstrukturen und unter Berücksichtigung der individuellen Einstellungen unterstützt werden kann (hierzu u.a. Duncombe und Armour 2004). Gerade mit Blick auf die Organisationsstrukturen, gilt es, Formate zu entwickeln, die Implementierungsbarrieren (hier insbesondere Zeit, Kosten, Zugang zu Fortbildungen) abbauen.

Unter Berücksichtigung der Befunde sowohl aus der quantitativen als auch der qualitativen Analyse lässt sich zusammenfassen, dass die durchgeführte Lehrkräfteprofessionalisierung eine Reihe der Gelingensbedingungen für erfolgreiche Kollaborationsprozesse erfüllt, was das Erfolgserlebnis sowie die wahrgenommene Wirksamkeit der Kollaboration fördert. Das hohe situationale Interesse unterstützt die Annahme, dass über das evaluierte Setting der kollaborativen Lehrkräfteprofessionalisierung Voraussetzungen für eine dauerhafte Veränderung der Einstellungen und Überzeugungen geschaffen werden.

Der Schwerpunkt des Beitrags liegt sowohl auf einer theoriegeleiteten Evaluation als auch auf einer explorativen Analyse. Daraus ergeben sich Implikationen für die Weiterbildungsforschung: Mit Blick auf die Ausgestaltung zukünftiger Evaluationsdesigns wären daher Motivationen auf state-Ebene, die das konkrete situationale Verhalten adressieren, im Modell umfangreicher zu berücksichtigen. Eine größere Bandbreite der Indikatoren sollte im Modell eingeschlossen werden. Um langfristige Veränderungsprozesse nach der Lehrkräfteprofessionalisierung zu erfassen, ist ein Follow-up-Design notwendig. Es ist zu analysieren, ob nach der Realisierung einer spezifischen Ausprägung des situationalen Interesses in der Fortbildung, auch eine Veränderung in den Einstellungen und Überzeugungen beobachtbar ist (Analyse von cut-off scores). Darüber hinaus ist es wünschenswert, das Konstrukt der Berufserfahrung der Lehrkräfte zu modellieren, um zu beantworten, welche Faktoren der Berufserfahrung den Transfer von neu gelernten pädagogischen Skills und Methoden 
in die eigene Lehrpraxis beeinflussen können. Ferner sind die im qualitativen Teil explorativ identifizierten Merkmale, die in der Fortbildung zu einer erfolgreichen Kollaboration beitragen können (z. B. gemeinsame Ergebniskotrolle, Aufgabenteilung und Perspektivübernahme), in zukünftigen Forschungen durch quantitative Methoden zu überprüfen.

Open Access Dieser Artikel wird unter der Creative Commons Namensnennung 4.0 International Lizenz veröffentlicht, welche die Nutzung, Vervielfältigung, Bearbeitung, Verbreitung und Wiedergabe in jeglichem Medium und Format erlaubt, sofern Sie den/die ursprünglichen Autor(en) und die Quelle ordnungsgemäß nennen, einen Link zur Creative Commons Lizenz beifügen und angeben, ob Änderungen vorgenommen wurden.

Die in diesem Artikel enthaltenen Bilder und sonstiges Drittmaterial unterliegen ebenfalls der genannten Creative Commons Lizenz, sofern sich aus der Abbildungslegende nichts anderes ergibt. Sofern das betreffende Material nicht unter der genannten Creative Commons Lizenz steht und die betreffende Handlung nicht nach gesetzlichen Vorschriften erlaubt ist, ist für die oben aufgeführten Weiterverwendungen des Materials die Einwilligung des jeweiligen Rechteinhabers einzuholen.

Weitere Details zur Lizenz entnehmen Sie bitte der Lizenzinformation auf http://creativecommons.org/ licenses/by/4.0/deed.de.

\section{Literatur}

Avalos, B. (2011). Teacher professional development in teaching and teacher education over ten years. Teaching and Teacher Education, 27(1), 10-20.

Baethge, M., \& Schiersmann, C. (1998). Prozessorientierte Weiterbildung - Perspektiven und Probleme eines neuen Paradigmas der Kompetenzentwicklung für die Arbeitswelt der Zukunft. Kompetenzentwicklung, 98, 15-87.

Beck, K. (2020). Ensuring content validity of psychological and educational tests-the role of experts. Frontline Learning Research, 8(6), 1-37. https://doi.org/10.14786/flr.v8i6.517.

Butler, D. L., \& Schnellert, L. (2012). Collaborative inquiry in teacher professional development. Teaching and teacher education, 28(8), 1206-1220.

Darling-Hammond, L., \& McLaughlin, M. W. (1995). Policies that support professional development in an era of reform. Phi Delta Kappan, 76, 597-604.

Davier, A. von, Zhu, M., \& Kyllonen, P. (2017). Methodology of educational measurement and assessment. Innovative assessment of collaboration. Cham: Springer. https://doi.org/10.1007/978-3-319-332611.

DIE [Deutsches Institut für Erwachsenenbildung] (Hrsg.). (2019). Curriculum Finanzielle Grundbildung. Bonn: DIE.

Duncombe, R., \& Armour, K. (2004). Collaborative professional learning: from theory to practice. Journal of in-Service Education, 30(1), 141-166. https://doi.org/10.1080/13674580400200230.

Europäische Kommission (2010). Towards more knowledge-based policy and practice in education and training.

Flake, R., Seyda, S., \& Werner, D. (2020). Weiterbildung während der Corona-Pandemie. https://idwonline.de/de/attachmentdata80095. Zugegriffen: 1. Okt. 2021.

Fögele, J., \& Mehren, R. (2015). Implementing geographical key concepts: design of a symbiotic teacher training course based on empirical and theoretical evidence. Review of International Geographical Education Online, 5(1), 56-76.

Friend, M., Cook, L., Hurley-Chamberlain, D., \& Shamberger, C. (2010). Co-teaching: an illustration of the complexity of collaboration in special education. Journal of Educational and Psychological Consultation, 20(1), 9-27.

Furnham, A., Jackson, C. J., \& Miller, T. (1999). Personality, learning style and work performance. Personality and Individual Differences, 27, 1113-1122.

Fussangel, K., \& Gräsel, C. (2012). Lehrerkooperation aus der Sicht der Bildungsforschung. In E. Baum, T. Idel \& H.H. Ullrich (Hrsg.), Kollegialität und Kooperation in der Schule. Wiesbaden: Springer VS. https://doi.org/10.1007/978-3-531-94284-1_2. 
Goldberg, L.R. (1993). The structure of phenotypic personality traits. American Psychologist, 48(1), 26-34.

Graesser, A., Fiore, S., Greiff, S., Andrews-Todd, J., Foltz, P., \& Hesse, F. (2018). Advancing the science of collaborative problem solving. Psychological Science in the Public Interest, 19(2), 59-92. https:// doi.org/10.1177/1529100618808244.

Griffin, G. (1983). Introduction: the work of staff development. In G. Griffin (Hrsg.), Staff development, eighty-second yearbook of the national society for the study of education. Chicago: University of Chicago Press.

Grotlüschen, A., \& Buddeberg, K. (Hrsg.). (2020). LEO 2018 - Leben mit geringer Literalität. Bielefeld: wbv.

Guskey, T. (2002). Professional development and teacher change. Teachers and Teaching, 8, 381-391.

Guskey, T., \& Sparks, D. (1996). Exploring the relationship between staff development and improvements in student learning. Journal of Staff Development, 17, 34-38.

Hayes, A. F. (2018). Introduction to mediation, moderation, and conditional process analysis: a regressionbased approach (2. Aufl.). New York: Guilford.

Hesse, F., Care, E., Buder, J., Sassenberg, K., \& Griffin, P. (2015). A framework for teachable collaborative problem solving skills. In E. Care \& P.E. Griffin (Hrsg.), Assessment and teaching of 21 st century skills: methods and approach (S. 37-56). Cham: Springer.

Holt, D. T., Armenakis, A. A., Feild, H. S., \& Harris, S. G. (2007). Readiness for organizational change: The systematic development of a scale. Journal of Applied Behavioral Science, 43(2), 232-255.

Hunsu, N. J., Adesope, O., \& Bayly, D. J. (2016). A meta-analysis of the effects of audience response systems (clicker-based technologies) on cognition and affect. Computers \& Education, 94, 102-119.

King, M., \& Newmann, F. (2001). Building school capacity through professional development: conceptual and empirical considerations. International Journal of Educational Management, 15(2), 86-93.

KMK (2011). Inklusive Bildung von Kindern und Jugendlichen mit Behinderungen in Schulen: Beschluss der Kultusministerkonferenz vom 20.10.2011. kmk.org. Zugegriffen: 1. Okt. 2021.

Koppel, I., \& Langer, S. (2020). Herausforderungen und Reaktionen in Zeiten des Social Distancing: Ein Blick in die Alphabetisierung und Grundbildung. weiter bilden, 28(4), 32-35.

Kullmann, H. (2016). Kollegiale Kooperation im Lehrerberuf. In M. Rothland (Hrsg.), Beruf Lehrer/ Lehrerin. Ein Studienbuch (S. 333-349). Münster: Waxmann.

Lewin, K. (1935). A dynamic theory of personality. New York: McGraw-Hill.

Löffler, C., \& Korfkamp, J. (2016). Handbuch zur Alphabetisierung und Grundbildung Erwachsener. Münster, New York: Waxmann.

Macleod, H. (2008). Games in learning. In M. Pivec \& M. Moretti (Hrsg.), Game-based learning: discover the pleasure of learning (S. 17-23). Lengerich: Pabst Science Publishers.

Mania, E., \& Tröster, M. (2014). Finanzielle Grundbildung - Ein Kompetenzmodell entsteht. Hessische Blätter für Volksbildung, 60, 136-145.

Mania, E., \& Tröster, M. (2015). Kompetenzmodell Finanzielle Grundbildung: Umgang mit Geld als Thema der Basisbildung. Magazin Erwachsenenbildung.at, 66(25), 72-81.

Mania, E., \& Tröster, M. (2018). Finanzen, Politik und Gesundheit als notwendige Inhalte der Grund-/ Basisbildung: Stand, Bedarfe und Herausforderungen. Magazin erwachsenenbildung.at, 33, 09-2-0910. http://nbn-resolving.de/urn:nbn:de:0111-pedocs- 154053.

Massenkeil, J., \& Rothland, M. (2016). Kollegiale Kooperation im Lehrerberuf. Überblick und Systematisierung aktueller Forschung. Schulpädagogik heute, 7(13).

Mayring, P. (2015). Qualitative Inhaltsanalyse: Grundlagen und Techniken (12. Aufl.). Weinheim, Basel: Beltz.

Neuweg, G. (2010). Fortbildung im Kontext eines phasenübergreifenden Gesamtkonzepts der Lehrerfortbildung. In F. Müller, A. Eichenberger, M. Lüders \& J. Mayr (Hrsg.), Lehrerinnen und Lehrer lernen - Konzepte und Befunde zur Lehrerfortbildung (S. 35-49). Münster: Waxmann.

OECD (2005). Teachers matters: attracting, developing and retaining effective teachers. Paris: OECD Publishing.

OECD (2009). Creating effective teaching and learning environments: First results from TALIS. Paris: OECD Publishing.

OECD (2017). PISA 2015 assessment and analytical framework: science, reading, mathematic, financial literacy and collaborative problem solving. Paris: OECD Publishing. https://doi.org/10.1787/ 9789264281820-en.

OECD (2019). TALIS 2018 results (volume I): teachers and school leaders as lifelong learners. Paris: OECD Publishing. https://doi.org/10.1787/1d0bc92a-en. 
OECD (2020). Back to the future of education: Four OECD scenarios for schooling. Educational research and innovation. Paris: OECD Publishing. https://doi.org/10.1787/178ef527-en.

Paeßens, J., \& Winther, E. (2021). Game design in financial literacy: exploring design patterns for a collaborative and inclusive serious game from different perspectives. In C. Aprea \& D. Ifenthaler (Hrsg.), Game-based learning across the disciplines (S. 43-59). Cham: Springer. https://doi.org/10.1007/9783-030-75142-5_3

Puteh, F., Kaliannan, M., \& Alam, N. (2015). Learning for professional development via peers: a system theory approach. Procedia-Social and Behavioral Sciences, 172, 88-95.

Rädiker, S., \& Kuckartz, U. (2019). Analyse qualitativer Daten mit MAXQDA: Text, Audio und Video (1. Aufl.). Wiesbaden: Springer. https://doi.org/10.1007/978-3-658-22095-2.

Sangmeister, J., Winther, E., Klotz, V., Bley, S., Kreuzer, C., \& Weber, S. (2018). Designing competence assessment in VET for a digital future. In D. Ifenthaler (Hrsg.), Digital workplace learning. Bridging formal and informal learning with digital technologies (S. 65-92). Cham: Springer.

Schmid, U., Goertz, L., \& Behrens, J. (2017). Monitor Digitale Bildung. Die Weiterbildung im digitalen Zeitalter. Gütersloh: Bertelsmann Stiftung.

Terhart, E. (2016). Personalauswahl, Personaleinsatz und Personalentwicklung an Schulen. In H. Altrichter \& K. Maag Merki (Hrsg.), Handbuch Neue Steuerung im Schulsystem (S. 279-299). Wiesbaden: Springer VS. https://doi.org/10.1007/978-3-531-92245-4_10.

Tröster, M., \& Bowien-Jansen, B. (2019a). Professionalisierung Finanzieller Grundbildung - Herausforderungen und Chancen eines Fortbildungskonzepts an der Schnittstelle von Erwachsenenbildung und Sozialer Arbeit. EPALE Blog Beitrag vom 29.08.2019.

Tröster, M., \& Bowien-Jansen, B. (2019b). Perspektive Praxis. Sensibel für Finanzielle Grundbildung: Studienmaterialien und Handlungsempfehlungen (1. Aufl.). Bielefeld: wbv Publikation.

Tröster, M., \& Schrader, J. (2016). Alphabetisierung, Grundbildung, Literalität: Begriffe, Konzepte, Perspektiven. In C. Löffler \& J. Korfkamp (Hrsg.), Handbuch zur Alphabetisierung und Grundbildung Erwachsener. UTB, (Bd. 8683, S. 42-58). Münster, New York: Waxmann.

Villegas-Reimers, E. (2003). Teacher professional development: an international review of the literature. Paris: UNESCO International Institute for Educational Planning.

Ward, P., \& O'Sullivan, M. (1998). Similarities and differences in pedagogy and content: 5 years later. Journal of Teaching in Physical Education, 17(2), 195-213. https://doi.org/10.1123/jtpe.17.2.195.

Warwas, J., Helm, C., \& Schadt, C. (2019). Unterstützendes Führungsverhalten schulischer Leitungskräfte für die Arbeit professioneller Lerngemeinschaften im Kollegium. Zeitschrift für Bildungsforschung, 9(1), 37-70. https://doi.org/10.1007/s35834-019-00230-w.

Winther, E., Paeßens, J., Tröster, M., \& Bowien-Jansen, B. (im Druck). Spielend lernen: MONETTO - Das Lernspiel für die Finanzielle Grundbildung. Hessische Blätter für Volksbildung, 2021(04). Erwachsenenbildung und Prekariat.

Zehner, F., Weis, M., Vogel, F., Leutner, D., \& Reiss, K. (2019). Kollaboratives Problemlösen in PISA 2015: Deutschland im Fokus. Zeitschrift für Erziehungswissenschaft, 22(3), 617-646.

Publisher's Note Springer Nature remains neutral with regard to jurisdictional claims in published maps and institutional affiliations. 